\title{
A predictive model for treatment response in patients with locally advanced esophageal squamous cell carcinoma after concurrent chemoradiotherapy: based on SUVmean and NLR
}

Chunsheng Wang ${ }^{1+}$, Kewei Zhao ${ }^{1,2+}$, Shanliang Hu${ }^{1}$, Yong Huang ${ }^{3}$, $\mathrm{Li} \mathrm{Ma}^{3}$, Yipeng Song ${ }^{1}$ and Minghuan $\mathrm{Li}^{2^{*}}$

\begin{abstract}
Background: We conducted this study to combine the mean standardized uptake value (SUVmean) and neutrophil to lymphocyte ratio (NLR) to establish a strong predictive model for patients with esophageal squamous cell carcinoma (ESCC) after concurrent chemoradiotherapy (CCRT).

Methods: We retrospectively analyzed 163 newly diagnosed ESCC patients treated with CCRT. Eighty patients (training set) were randomly selected to generate cut-off SUVmean and NLR values by receiver operating characteristic (ROC) curve analysis and to establish a predictive model by using the independent predictors of treatment outcomes. Then, we evaluated the performance of the prediction model regarding treatment outcomes in the testing set $(n=83)$ and in all sets.

Results: A high SUVmean (> 5.81) and high NLR (> 2.42) at diagnosis were associated with unfavorable treatment outcomes in patients with ESCC. The prediction model had a better performance than the simple parameters $(p<0.05)$. With a cut-off value of 0.77 , the prediction model significantly improved the specificity and positive predictive value for treatment response (88.9 and $92.1 \%$ in the training set, 95.8 and $97.1 \%$ in the testing set, and 92.2 and $91.8 \%$ in all sets, respectively).
\end{abstract}

Conclusions: The pretreatment SUVmean and NLR were independent predictors of treatment response in ESCC patients treated with CCRT. The predictive model was constructed based on these two parameters and provides a highly accurate tool for predicting patient outcomes.

Keywords: Esophageal squamous cell carcinoma, Predictive model, Treatment response, Concurrent chemoradiotherapy, SUVmean, NLR

\footnotetext{
* Correspondence: sd Imh@sina.com

${ }^{+}$Chunsheng Wang and Kewei Zhao contributed equally to this work. ${ }^{2}$ Department of Radiation Oncology, Shandong Cancer Hospital and Institute, Shandong University, 440 Jiyan Road, Jinan 250117, Shandong, People's Republic of China

Full list of author information is available at the end of the article
}

(c) The Author(s). 2020 Open Access This article is licensed under a Creative Commons Attribution 4.0 International License, which permits use, sharing, adaptation, distribution and reproduction in any medium or format, as long as you give appropriate credit to the original author(s) and the source, provide a link to the Creative Commons licence, and indicate if changes were made. The images or other third party material in this article are included in the article's Creative Commons licence, unless indicated otherwise in a credit line to the material. If material is not included in the article's Creative Commons licence and your intended use is not permitted by statutory regulation or exceeds the permitted use, you will need to obtain permission directly from the copyright holder. To view a copy of this licence, visit http://creativecommons.org/licenses/by/4.0/ The Creative Commons Public Domain Dedication waiver (http://creativecommons.org/publicdomain/zero/1.0/) applies to the data made available in this article, unless otherwise stated in a credit line to the data. 


\section{Background}

Concurrent chemoradiotherapy (CCRT) has been established as the standard treatment for locally advanced inoperable esophageal cancer (EC) patients, according to the phase III intergroup RTOG 85-01 trial [1]. Although CCRT improved local control and overall survival compared with radiotherapy alone, the treatment outcomes of CCRT varied widely. According to data in the literature, the overall response rate (ORR) to CCRT in patients with esophageal cancer ranges from 53.3 to $98.3 \%$ [2-4]. We can improve this rate by setting individualized treatment strategies and intensities for different subgroups of patients. However, it is quite difficult to balance the risks of complications and treatment benefits without knowing the effects before treatment. Therefore, the early prediction of the tumor response before treatment may benefit this heterogeneous group of patients.

${ }^{18}$ F-fluorodeoxy-glucose Positron emission tomography/computed tomography $\left({ }^{18}\right.$ F-FDG PET/CT) allows visualization of the high glucose utilization in tumor tissue, based on the assumption that cancer cells generally exhibit a higher level of glycolytic activity than healthy cells. A semiquantitative parameter derived from FDG-PET, maximum standardized uptake values (SUVmax), has been widely used to quantitate the metabolic activity of tumors [5-7]. However, SUVmax is measured on a single voxel and may not reflect the metabolism within the whole tumor $[8,9]$. Mean of standardized uptake values (SUVmean), another metabolic parameter, is subsequently measured to calculate the average SUVs above a threshold (SUV > 2.5), which might reflect the metabolic burden of the entire tumor as opposed to that of a single point $[10,11]$. Previous studies on different solid tumors have shown a correlation between SUVmean and tumor treatment outcomes [1214]. On the other hand, recent studies have revealed that cancer-related inflammation plays an important role in cancer progression and metastasis [15-17]. Neutrophil-tolymphocyte ratio (NLR), as a systemic inflammatory marker, has been reported to be associated with tumor response and prognosis in esophageal cancer [18, 19]. However, these studies mainly explored the predictive effect of NLR in patients undergoing surgery, researches focused on the role of NLR in predicting tumor response in nonsurgically patients have been rarely reported [20, 21].

Hence, in the present study, we attempted to establish a prediction model for the treatment effects of CCRT for esophageal cancer patients based on two aspects: the abnormal glucose metabolism of tumor cells and the anti-tumor immune response of the host.

\section{Methods}

\section{Patients}

We retrospectively analyzed 163 locally advanced ESCC patients who were treated with CCRT in shandong cancer hospital between January 2011 to December 2017. Patients were included if they had a Karnofsky performance scale (KPS) score $\geq 70$ and had ESCC confirmed by histopathological analysis. They also need fulfilled the following criteria: (1) available complete clinical information;(2) completed PET/CT examination and routine blood test one week before any treatment;(3) No history of other malignancy or secondary primary tumor;(4) without any acute infections or any hematologic disease and autoimmune diseases; (5) locally advanced disease based on the 7th edition of the American Joint Committee on Cancer guidelines (AJCC7th edition). Of the 163 patients, 80 patients were randomly assigned to the training set using a computer program, while the remaining patients ( $n=83$ ) were assigned to the testing set. The ethics committee of Shandong Cancer Hospital and Institute approved the study. And informed consent was exempted due to the retrospective nature of the study.

\section{Treatment protocols and response assessment}

All patients received intensity-modulated radiation therapy with a total dose of 50-64 Gy administered once daily with a standard fractionation (ie 1.8 or $2.0 \mathrm{~Gy} / \mathrm{frac}-$ tions, 25-32 fractions, 5 days/week). Chemotherapy was administered simultaneously with the initial radiotherapy on Day 1. cisplatin $(75 \mathrm{mg} / \mathrm{m} 2)$ was administered by iv on Day 1 and 5-Fluorouracil $(700 \mathrm{mg} / \mathrm{m} 2)$ was administered intravenously (iv) continuous infusion over $24 \mathrm{~h}$ daily on Days $1-4$. Cycled every 28 days for $2-4$ cycles for 2 cycles with radiation followed by 2 cycles without radiation. Patients were asked to visit the clinic within 2-4 weeks after completion of all therapies. Contrastenhanced computed tomography scan was performed 24 weeks after the end of treatment for evaluate treatment response. The tumor response was assessment based on evaluation criteria in solid tumors (RECIST) Version 1. 1. A CR was defined as no evidence of disease and tumor marker measurements within normal ranges for at least 2 weeks. Partial response (PR) was defined as a decrease in the lesion as measured bidimensionally by at least $30 \%$ with no signs of either new lesions or progression of any existing lesions. Progressive disease (PD) was defined as an increase of at least $20 \%$ in a lesion as measured bidimensionally, the appearance of any new lesions, or a previously eradicated lesion reappearing. Stable disease (SD) was defined as a tumor response that did not fulfill the PR criteria but exceeded the PD criteria. A primary tumor response that fulfilled the CR criteria and PR criteria was defined as objective response (OR) $(\mathrm{OR}=\mathrm{CR}+\mathrm{PR})$, and the other was defined as Non-OR.

\section{PET/CT scanning and image analysis}

$\mathrm{PET} / \mathrm{CT}$ scanning was performed before any anti-cancer treatment with an advanced PET/CT scanner (Discovery 
LS, GE Healthcare). Before undergoing PET/CT scans, all patients were asked to fast for at least $6 \mathrm{~h}$ and have a blood glucose level $\leq 11.1 \mathrm{mmol} / \mathrm{L}$. Then each of they were injected into $5.18 \mathrm{MBq} / \mathrm{kg}$ of ${ }^{18} \mathrm{~F}$-FDG.One hour later, a whole-body PET and CT scans from top of the skull to the proximal thigh were initiated for $5 \mathrm{~min}$ per field of view, each covering $14.5 \mathrm{~cm}$, with an axial sampling at $4.25 \mathrm{~mm}$ per slice. Then use the ordered-subset expectation maximization algorithm to reconstructed PET data sets by CT-derived attenuation correction. The attenuation-corrected PET images, CT images, as well as fused PET/CT images were displayed as coronal, sagittal, and axial slices on the Xeleris workstation (GE Healthcare). Measurements were obtained by two nuclear medicine physicians with at least 10 of experience and who were unaware of the patients' clinical and pathological results. The standard uptake values (SUVs) were obtained with the contour threshold method and were based on a region of interest (ROI). An SUV threshold of 2.5 was used to define the ROI boundaries, which has been widely approved. A volumetric ROI was placed around the outline of the primary tumor on the axial PET/CT images using semiautomatic software. The ROI borders were manually adjusted by visual inspection of the primary tumor to avoid overlapping with adjacent FDG-avid structures. The SUVmean value is the average of the metabolic activity in the ROIs and was automatically calculated by the software. Metabolic tumor volume (MTV) was defined as the volume of the part of the primary lesion that was obtained using the cutoff (SUV $\geq$ 2.5). Total lesion glycolysis (TLG) was calculated by multiplying SUVmean by MTV.

\section{Laboratory data}

Venous blood samples were collected between 6 and 9 am 1 week before any anti-cancer treatment, and both the peripheral neutrophils and lymphocytes were counted by Sysmex XT-2000i Automated Hematology Analyzer (GMI, MN, USA). The peripheral NLR was defined as the absolute neutrophil count divided by the absolute lymphocyte count.

\section{Statistical analysis}

The selection of cut-off values for the baseline SUVmean and peripheral NLR was determined using receiver operating characteristic (ROC) curve analysis in the training set $(n=80)$. The correlations between SUVmean and the NLR and clinicopathological parameters were assessed by Fisher's exact or chi-squared tests in the training set. Spearman's correlation coefficient test was used to estimate the correlation between the SUVmean and NLR level. Univariate and multivariate logistic regression analyses were performed in the training set to identify the independent predictors for tumor response. The independent predictive factors were used to establish the prediction model for treatment response to CCRT in esophageal cancer patients and to construct the regression equation for calculating the model prediction value (Y-value). Delong's test was used to analyze the area under the curve (AUC) of the ROC curves and to compare the accuracy of each prediction index (Yvalue, SUVmean, and NLR). The Y-value of each patient was calculated in the testing set by using the regression equation. The sensitivity and specificity of the predictive model were evaluated in the testing set and in all patients. SPSS 22.0 program (SPSS Inc., Chicago, IL, USA) and MedCalc program (Version 18.11) were used to conduct these analyses, a two-sided $p$-value less than 0.05 was considered statistical significance.

\section{Results}

\section{Patient characteristics}

A total of 163 newly diagnosed ESCC patients treated with CCRT were retrospectively analyzed, including 127 (77.9\%) males and 36 (22.1\%) females. The median age was 65 years (range: 39-90 years). In our sample, there were slightly more patients who had a history of smoking (56.4\%) than those who had never smoked; a similar distribution was observed for patients with a history of alcohol consumption. Of the 163 patients with ESCC, 12 (7.4\%) tumors were located in the cervical, 50 (30.7\%) were located in the upper thoracic, 75 (46.0\%) were located in the mid-thoracic, and 26 (16.0\%) were located in the lower thoracic esophagus. Additionally, most of the patients had stage III disease (122, 74.8\%), whereas 41 (25.2\%) had stage II disease. 112 (68.7\%) patients in the OR and 51 (31.3\%) non-OR groups were and, respectively, with an overall ORR of $68.7 \%$ (Table 1 ).

\section{Comparison of PET parameters}

We conducted a preliminary analysis of the predictive effect of PET parameters on predicting the treatment response of CCRT in esophageal cancer by performing a receiver operating characteristic (ROC) curve. The results show that SUVmean has the highest predicting accuracy. The AUCs of ROC curve of SUVmean, NLR, MTV and TLG were 0.732 (95\% CI: 0.657-0.798),0.652 (95\% CI: 0.574-0.725),0.628 (95\% CI: 0.549-0.703), and 0.668 (95\% CI: 0.590-0.739), respectively (supplement Table 1). The $\triangle A U C$ (calculated by subtracting the AUCs of the other three PET parameters from that of SUVmean respectively) between SUVmax, MTV and SUVmean were 0.0797 and 0.1030 , the difference was statistically significant $(p=0.0019, p=0.0021$, respectively). The $\triangle A U C$ between TLG and SUVmean has differential tendencies, but no statistical significance $(\triangle \mathrm{AUC}=0.0642, p=0.00746)$ (Fig. 1). 
Table 1 Baseline clinical characteristics of patients

\begin{tabular}{|c|c|c|c|}
\hline Characteristics & $\begin{array}{l}\text { All cases } \\
(n=163)\end{array}$ & $\begin{array}{l}\text { Training set } \\
(n=80)\end{array}$ & $\begin{array}{l}\text { Testing set } \\
(n=83)\end{array}$ \\
\hline \multicolumn{4}{|l|}{ Age (years) } \\
\hline median & 65 & 66 & 65 \\
\hline range & $39-90$ & $39-90$ & $44-84$ \\
\hline \multicolumn{4}{|l|}{ Sex } \\
\hline Male & $127(77.9)$ & 58 & 69 \\
\hline Female & $36(22.1)$ & 22 & 14 \\
\hline \multicolumn{4}{|l|}{ Smoking history } \\
\hline Yes & $92(56.4)$ & 41 & 51 \\
\hline No & 71 (43.6) & 39 & 32 \\
\hline \multicolumn{4}{|l|}{ Drinking history } \\
\hline Yes & $86(56.4)$ & 41 & 45 \\
\hline No & 77 (43.6) & 39 & 38 \\
\hline \multicolumn{4}{|l|}{ T stage } \\
\hline $1-3$ & $128(78.5)$ & 63 & 65 \\
\hline 4 & 35 (21.5) & 17 & 18 \\
\hline \multicolumn{4}{|l|}{$\mathrm{N}$ stage } \\
\hline 0 & $137(84.0)$ & 63 & 74 \\
\hline $1-3$ & $26(16.0)$ & 17 & 9 \\
\hline \multicolumn{4}{|l|}{ Tumor stage } \\
\hline$\|$ & $41(25.2)$ & 20 & 21 \\
\hline III & $122(74.8)$ & 60 & 62 \\
\hline \multicolumn{4}{|l|}{ Tumor location } \\
\hline Cervical & $12(7.4)$ & 5 & 7 \\
\hline Upper thoracic & $50(30.7)$ & 27 & 23 \\
\hline Mid-thoracic & $75(46.0)$ & 36 & 39 \\
\hline Lower thoracic & $26(16.0)$ & 12 & 14 \\
\hline \multicolumn{4}{|l|}{ Tumor response } \\
\hline OR & $112(68.7)$ & 56 & 56 \\
\hline Non-OR & $51(31.3)$ & 24 & 27 \\
\hline
\end{tabular}

Univariate and multivariate analyses in the training set

To understand the prognostic values of SUVmean and NLR, we determined the optimal cut-off values by receiver operating characteristic (ROC) curve analysis in the training set. The AUCs of the SUVmean and NLR were 0.731 (95\% CI: $0.620-0.824)$ and 0.686 (95\% CI: $0.573-0.785$ ) with optimal cut-off values of 5.81 (sensitivity: $75.0 \%$, specificity: $69.6 \%$ ) and 2.42 (sensitivity: 75.0\%, specificity: 66.1\%), respectively (Fig. 2a). The baseline data of the patients in different SUVmean and NLR groups are summarized in Table 2. We examined the relationship between SUVmean and NLR. The result showed that there was a significant, however, quite weak positive correlation between SUVmean and NLR ( $\mathrm{r}=$ $0.289, P=0.009$; Fig. 3$)$. The univariate analysis revealed that tumor stage $(P=0.020)$, SUVmean $(P<0.001)$ and
NLR $(P=0.001)$ were prognostic factors for OR. None of the other parameters (i.e., age, sex, smoking history, drinking history, $\mathrm{T}$ stage, $\mathrm{N}$ stage, and tumor location) showed significant differences among groups (Table 3). Subsequently, the multivariate analysis revealed that a low tumor stage $(\mathrm{HR}=10.92 ; 95 \% \mathrm{CI}, 1.17-102.12 ; P=$ 0.036), low NLR ( $\mathrm{HR}=7.17$; 95\% CI: 2.12-24.20), and low tumor SUVmean $(\mathrm{HR}=3.95$; 95\% CI, 1.16-13.47; $P=0.028$ ) were significant independent predictors for good treatment response.

\section{Construction and validation of the prediction model}

Based on the multivariate analysis of the training set, a logistic regression model was generated using tumor stage, the SUVmean value and the NLR value. The analysis results of the model are shown in Table 4. The logistic regression equation of the prediction model is as follows: $\mathrm{Y}=1 /\left[1+\mathrm{e}^{-\left(7.849-3.728 * \mathrm{x}_{1}-0.449 * \mathrm{x}_{2}-0.338 * \mathrm{x}_{3}\right)}\right]$. In the formula, $\mathrm{x}_{1}$ is the score of the tumor stage (stage II: 0; stage III: 1 ), $\mathrm{x}_{2}$ is the NLR value, and $\mathrm{x}_{3}$ is the SUVmean value. The Yvalues of each patient were calculated according to the predictive model equation. ROC analysis was then performed to validate the final model (including tumor stage, SUVmean, NLR) in the training set; the resulting AUC was 0.826 (95\% CI: $0.725-0.902)$ with a cut-off value of 0.77. Comparing the AUCs of the predictive model with that of the "single model" (SUVmean or NLR) by Delong's test, a significant $P$-value was observed $(P=0.048$, and $P=$ 0.012 , respectively) (Fig. 2 a). The same procedure was then performed in the testing set and in all patients, and the results show that the full model also had a better performance than the "simple model" in both samples (Table 5). Subsequently, the patients in the testing set and all sets were dichotomized based on the cut-off values of the SUVmean, NLR and Y-values obtained in the training set. Each of the variables was then used to predict the treatment response of the three samples. The sensitivity, specificity, positive predictive value, and negative predictive value of each variable for predicting treatment outcomes were evaluated (Fig. 4). With a cut-off value of 0.77, the combined model significantly improved the specificity and positive predictive value. The specificity and positive predictive value increased to 95.8 and $97.1 \%$ in the training set, to 88.9 and $92.1 \%$ in the testing set and to 92.2 and $94.5 \%$ in all sets, respectively. However, the sensitivity and negative predictive value of the combined model did not seem to be better than those of the SUVmean or NLR values were. Figure 5 was a typical case presentation with PET image parameter.

\section{Discussion}

In the present study, we demonstrated that the pretreatment SUVmean and NLR were independent predictive 


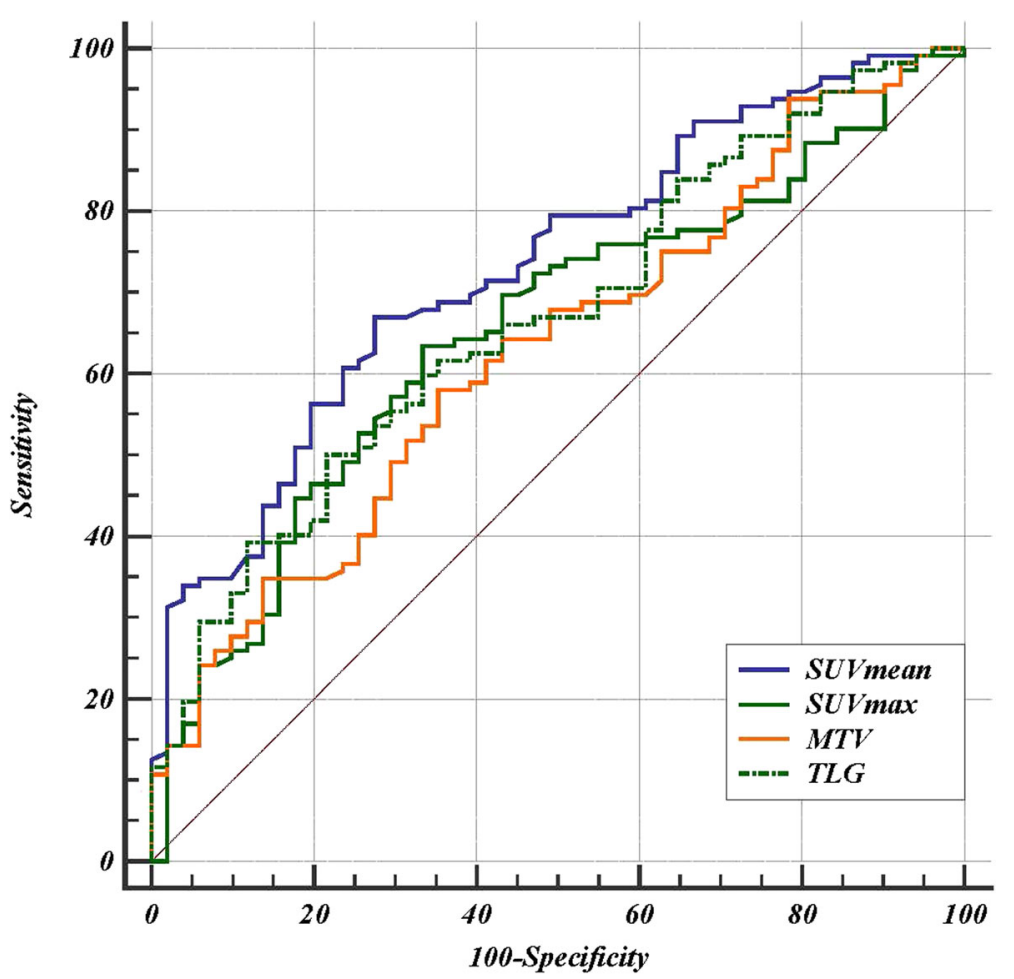

Fig. 1 ROC curves of PET parameters

factors of treatment response to CCRT in patients with locally advanced esophageal cancer. Moreover, we developed a novel predictive model based on the pretreatment SUVmean and NLR values. This model had a good performance and may serve as an accurate and convenient tool for predicting the treatment response of patients and could make contributions to improving treatment outcomes and prognoses. To the best of our knowledge, this is the first predictive model for treatment response to CCRT in patients with locally advanced esophageal cancer that takes into account both tumor metabolic activity and host immunity.
${ }^{18}$ F-FDG PET/CT, which reflects glucose metabolism, has been widely applied in the management of oncological patients. In addition to detecting the primary tumor, this imaging modality also plays an important role in treatment response prediction. The semiquantitative data derived from such imaging, such as SUVmax and SUVmean as well as MTV and TLG, have been used for tumor response prediction in various cancers, including EC. Recent studies have shown that SUVmean provides a better picture of whole-tumor metabolic activity than SUVmax, which may only represent the single pixel of greatest metabolic activity within a tumor [10-13].
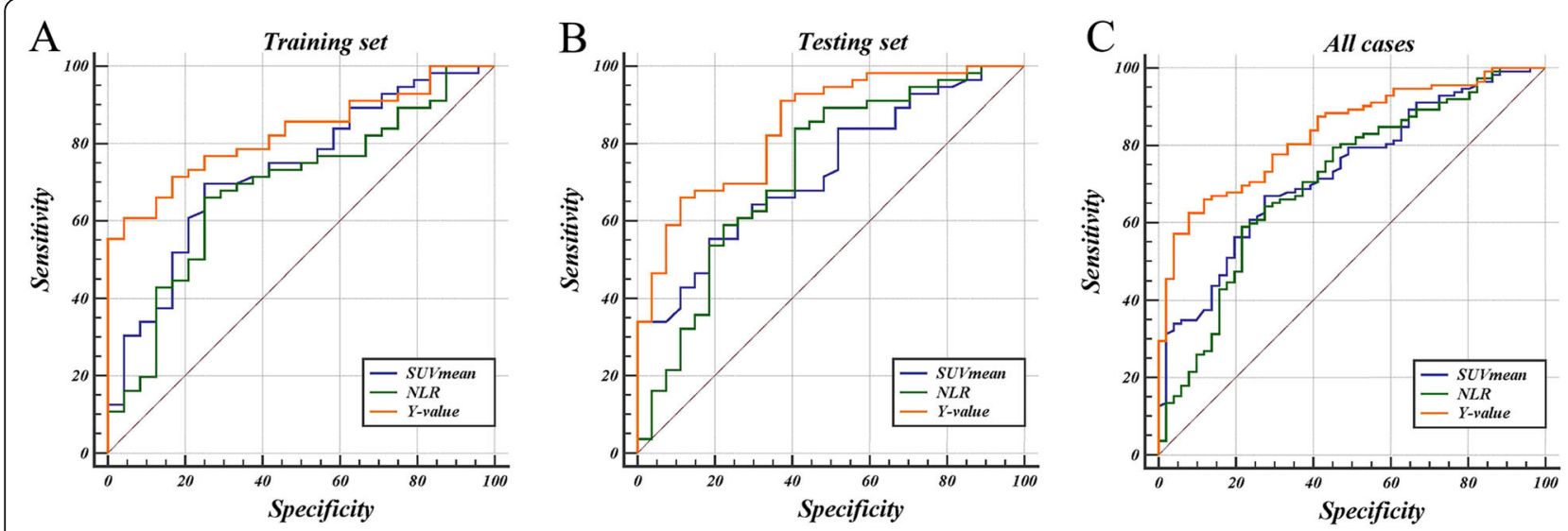

Fig. 2 ROC curves of three variables for treatment prediction in different set samples 
Table 2 Baseline data between different SUV mean and NLR groups in training set

\begin{tabular}{|c|c|c|c|c|c|c|}
\hline \multirow[t]{2}{*}{ Characteristics } & \multicolumn{3}{|c|}{$\underline{\text { SUVmean }}$} & \multicolumn{3}{|l|}{$\underline{N L R}$} \\
\hline & $\leq 5.81$ & $>5.81$ & $p$ & $\leq 2.42$ & $>2.42$ & $p$ \\
\hline \multicolumn{7}{|l|}{ Age (years) } \\
\hline$<60$ & 19 & 8 & 0.069 & 17 & 10 & 0.238 \\
\hline$\geq 60$ & 26 & 27 & & 26 & 27 & \\
\hline \multicolumn{7}{|l|}{ Sex } \\
\hline Male & 32 & 26 & 0.752 & 30 & 28 & 0.555 \\
\hline Female & 13 & 9 & & 13 & 9 & \\
\hline \multicolumn{7}{|l|}{ Smoking history } \\
\hline Yes & 22 & 19 & 0.632 & 22 & 19 & 0.987 \\
\hline No & 23 & 16 & & 21 & 18 & \\
\hline \multicolumn{7}{|l|}{ Drinking history } \\
\hline Yes & 23 & 18 & 0.978 & 23 & 19 & 0.666 \\
\hline No & 22 & 17 & & 20 & 18 & \\
\hline \multicolumn{7}{|l|}{ T stage } \\
\hline $1-3$ & 36 & 27 & 0.757 & 32 & 31 & 0.307 \\
\hline 4 & 9 & 8 & & 11 & 6 & \\
\hline \multicolumn{7}{|l|}{$\mathrm{N}$ stage } \\
\hline 0 & 13 & 4 & 0.058 & 10 & 7 & 0.636 \\
\hline $1-3$ & 32 & 31 & & 33 & 30 & \\
\hline \multicolumn{7}{|l|}{ Tumor stage } \\
\hline$\|$ & 18 & 2 & $<0.001$ & 10 & 10 & 0.698 \\
\hline III & 27 & 33 & & 33 & 27 & \\
\hline \multicolumn{7}{|l|}{ Tumor location } \\
\hline Cervical & 3 & 2 & 0.746 & 4 & 1 & 0.323 \\
\hline Upper thoracic & 16 & 11 & & 15 & 12 & \\
\hline Mid-thoracic & 18 & 18 & & 20 & 16 & \\
\hline Lower thoracic & 8 & 4 & & 4 & 8 & \\
\hline \multicolumn{7}{|l|}{ Tumor response } \\
\hline OR & 39 & 17 & $<0.001$ & 6 & 18 & 0.001 \\
\hline Non-OR & 6 & 18 & & 37 & 19 & \\
\hline \multicolumn{7}{|l|}{ SUVmean } \\
\hline$\leq 5.81$ & - & - & - & 28 & 17 & 0.085 \\
\hline$>5.81$ & - & - & & 15 & 20 & \\
\hline \multicolumn{7}{|l|}{ NLR } \\
\hline$\leq 2.42$ & 17 & 20 & 0.85 & - & - & - \\
\hline$>2.42$ & 28 & 15 & & - & - & \\
\hline
\end{tabular}

For example, a previous retrospective study of locally advanced cervical cancer revealed that patients with high SUVmean values were associated with poor posttreatment responses to definitive chemoradiotherapy [12]. Our results similar with this finding; patients with a low SUVmean $(\leq 5.81)$ are more likely to have a good tumor response than those with a high SUVmean (> 5.81). Our research suggests that SUVmean has higher diagnostic accuracy than SUVmax, MTV and TLG in predicting the response to treatment,it is an independent predictor of treatment response in locally advanced esophageal cancer patients treated with CCRT.

Cancer-related inflammation affects tumor proliferation and survival, angiogenesis, metastasis, and response to treatment [15-17]. Indeed, inflammation is now considered one of the hallmarks of cancer. The precise mechanism of these correlations is not yet clear, but there are some hypotheses on this issue. On the one hand, neutrophils contain and secrete a large number of inflammatory factors that directly contribute to tumor angiogenesis, vascular formation, growth and metastasis [15-17]. In addition, the circulating neutrophils could act as a surrogate for tumor-associated neutrophils, which act as adhesive adapters between circulating tumor cells and the metastatic target and play an important role in tumor angiogenesis and growth by secreting vascular endothelial growth factor and matrix metalloproteinase $[15,17]$. On the other hand, lymphocytes possess an anti-tumor effect by inducing tumor cell apoptosis and mediating antibody-dependent cell-mediated cytotoxicity [22-24]. Moreover, memory T-cells are considered to have a crucial role in carcinogenesis [25]. Based on the contributions of inflammation to carcinogenesis and tumor progression, the prognostic value of NLR has been investigated in various types of cancers [18-20, 26-28]. All of the previous studies came to the conclusion that an elevated NLR is associated with poor outcomes. However, evidence for the prognostic role of NLR in esophageal cancer is relatively controversial. Kosumi $\mathrm{K}$ et al. [29] investigated the relationship between the preoperative NLR and prognosis in 238 patients with esophageal squamous cell carcinoma. The results showed that with a median of 1.94 as the cut-off value, the high-NLR group had a 3-year cancer-specific survival rate and 3-year survival rate of 81.1 and $82.3 \%$, respectively, which were significantly higher than those in the low-NLR group (59.8 and 68.4\%, respectively). A high preoperative NLR was significantly associated with short overall survival. Another study found that an elevated preoperative NLR $(\geq 5$. 0$)$ level can be used as an independent prognostic indicator to predict recurrence and death after esophagectomy. The patients with elevated NLR levels had poor cancer-free survival and overall survival [18]. However, on the contrary, some investigators have documented that the pretreatment NLR did not predict the outcomes of patients treated with esophagectomy [30,31]. These studies focused primarily on the long-term survival of patients undergoing surgery for esophageal cancer, and the NLR cut-off values have not yet been fixed, varying from 1.95 to 5.0. The predictive value of NLR for treatment outcomes in patients with locally advanced esophageal cancer receiving CCRT has rarely been reported. Yoo EJ et al. retrospectively analyzed 


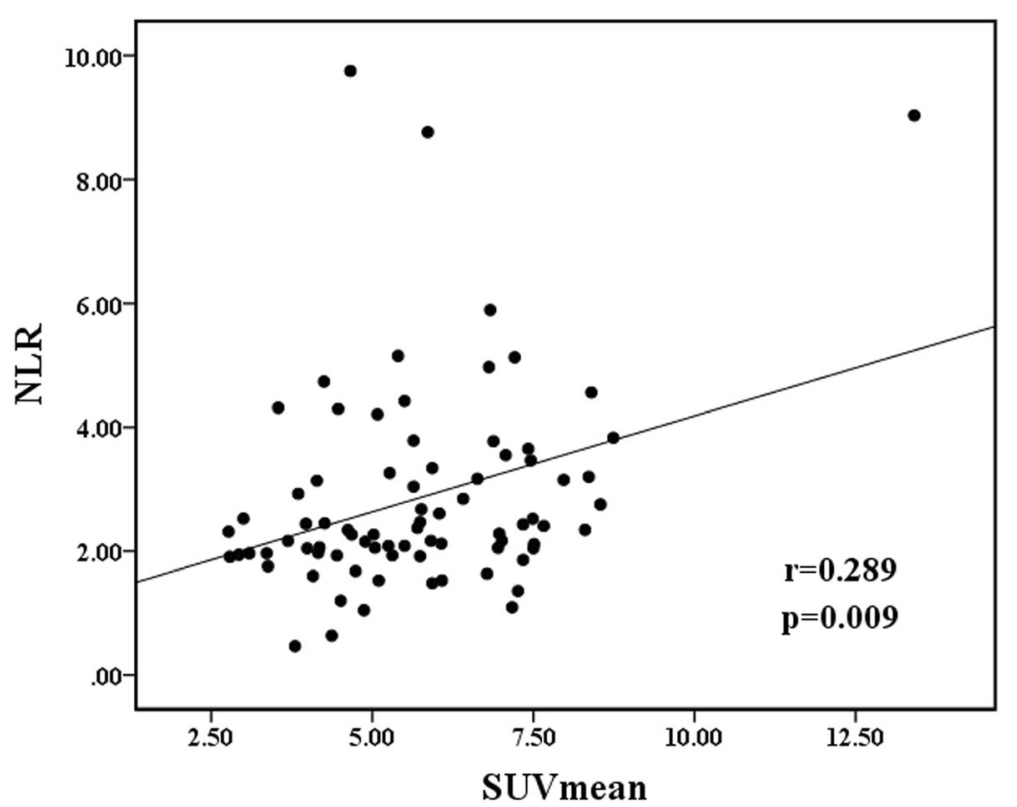

Fig. 3 Spearman's correlation analyses indicating a significant correlation between that SUVmean and NLR $(r=0.289, p=0.009)$

138 patients with locally advanced esophageal cancer and concluded that an elevated NLR was an independent predictor of poor outcomes for patients treated with CCRT [20]. This result is similar to our study. In our study, we use a ROC curve to determine the cut-off value of NLR, which balanced sensitivity and specificity. The results of this study indicate that NLR is an independent predictor of treatment response in patients undergoing CCRT and that patients with a high NLR $(>2.42)$ are more likely to have a poor treatment outcome than patients with a low NLR. The similarities of our studies stress the importance of further research on NLR for predicting the treatment outcomes of CCRT.

PET parameters represent an estimate of glucose metabolism in the entire tumor lesion, and hematological inflammation parameters reflect the host's anti-tumor immunological response. The combined evaluation of these two factors may provide complementary information and may be highly effective for predicting the outcomes and prognosis of patients. There are some previous reports that identified the relationship between PET parameters and hematological inflammation parameters. For example,

Table 3 Univariate and multivariate analyses for tumor response in training set (OR and non-OR)

\begin{tabular}{|c|c|c|c|c|c|c|c|}
\hline \multirow[t]{2}{*}{ variable } & \multirow[t]{2}{*}{ Categories } & \multicolumn{3}{|c|}{ Univariate analysis } & \multicolumn{3}{|c|}{ Multivariate analysis } \\
\hline & & $\mathrm{HR}$ & $95 \% \mathrm{Cl}$ & $p$ & $\mathrm{HR}$ & $95 \% \mathrm{Cl}$ & $p$ \\
\hline Age & $<60$ versus $\geq 60$ & 0.556 & $0.190-1.621$ & 0.282 & & & \\
\hline Sex & Male versus Female & 0.833 & $0.280-2.480$ & 0.743 & & & \\
\hline Smoking history & Yes versus No & 1.074 & $0.413-2.795$ & 0.884 & & & \\
\hline Drinking history & Yes versus No & 1.074 & $0.413-2.795$ & 0.884 & & & \\
\hline T stage & $\mathrm{T} 1-3$ versus $\mathrm{T} 4$ & 0.733 & $0.236-2.282$ & 0.592 & & & \\
\hline $\mathrm{N}$ stage & T0 versus T1-3 & 0.273 & $0.057-1.309$ & 0.105 & & & \\
\hline Tumor stage & II versus III & 11.81 & $1.48-94.27$ & 0.020 & 10.92 & $1.17-102.12$ & 0.036 \\
\hline \multirow[t]{4}{*}{ Tumor location } & Cervical & 2.000 & $0.201-19.914$ & 0.554 & & & \\
\hline & Upper thoracic & 1.429 & $0.473-4.313$ & 0.527 & & & \\
\hline & Mid-thoracic & reference & - & - & & & \\
\hline & Lower thoracic & 1.000 & $0.250-3.998$ & 1.000 & & & \\
\hline NLR & $\leq 2.42$ versus $>2.42$ & 5.84 & $1.99-17.15$ & 0.001 & 7.17 & $2.12-24.20$ & 0.002 \\
\hline SUVmean & $\leq 5.81$ versus $>5.81$ & 6.88 & $2.33-20.38$ & $<0.001$ & 3.95 & $1.16-13.47$ & 0.028 \\
\hline
\end{tabular}


Table 4 Logistic regression models fitted on training set samples

\begin{tabular}{llllllll}
\hline Variable & $\mathrm{B}$ & $\mathrm{S} . \mathrm{E}$. & Wald & $\mathrm{df}$ & $\mathrm{p}$ & $\mathrm{OR}$ & $95 \% \mathrm{Cl}$ \\
\hline Tumor stage & -3.728 & 1.873 & 3.960 & 1 & 0.047 & 0.024 & $0.001-0.945$ \\
NLR & -0.449 & 0.209 & 4.596 & 1 & 0.032 & 0.638 & $0.423-0.962$ \\
SUVmean & -0.338 & 0.194 & 4.008 & 1 & 0.045 & 0.678 & $0.464-0.992$ \\
\hline
\end{tabular}

Fujii $\mathrm{T}$ et al. showed a significant positive correlation between the NLR and SUVmax values in 143 patients with invasive ductal breast cancer [32]. A similar study conducted by Jeong $\mathrm{E}$ et al. [33] with 1034 newly diagnosed nonsmall-cell lung cancer patients investigated the relationship between SUVmax and circulating blood cell-based parameters. A weak but statistically significant correlation was found between SUVmax and NLR. Furthermore, several studies have demonstrated a direct association between metabolic tumor volume (MTV) and NLR [34-36]. In our present study, we determined that SUVmean also had a positive correlation with NLR. This result was consistent with previously reported findings [37]. However, the precise mechanism behind these correlations is complicated and is currently under investigation, but certain opinions may be useful for interpreting the mechanism. One possible opinion may be that inflammatory cells, such as lymphocytes, neutrophils, and macrophages, infiltrate the malignant lesions to increase the intake of FDG to reflect more energy consumption [38]. Another potential explanation may involve inflammation-induced angiogenesis. Hypoxia and persistent neovascularization are core features of the tumor microenvironment. Hypoxia in the tumor microenvironment promotes the secretion of angiogenic factors by increasing the number of inflammatory cells, resulting in the production of a large number of new blood vessels, which is then accompanied by an increase in tumor FDG uptake

Table 5 Comparison of ROC curves

\begin{tabular}{llllll}
\hline Variable & AUC & SE. & $95 \% \mathrm{Cl}$ & ${ }^{\mathrm{a} A U C}$ & $p$-value \\
\hline $\begin{array}{llllll}\text { Training set } \\
\text { SUVmean }\end{array}$ & 0.731 & 0.0608 & $0.620-0.824$ & 0.0952 & $\mathbf{0 . 0 4 8 5}$ \\
NLR & 0.686 & 0.0649 & $0.573-0.785$ & 0.1400 & $\mathbf{0 . 0 1 2 2}$ \\
Y-value & 0.826 & 0.0449 & $0.725-0.902$ & - & - \\
Testing set & & & & & \\
SUVmean & 0.728 & 0.0562 & $0.619-0.820$ & 0.117 & $\mathbf{0 . 0 1 6 7}$ \\
NLR & 0.730 & 0.0628 & $0.622-0.822$ & 0.115 & $\mathbf{0 . 0 2 0 4}$ \\
Y-value & 0.845 & 0.0443 & $0.749-0.915$ & - & - \\
All case & & & & & \\
SUVmean & 0.732 & 0.0406 & $0.657-0.798$ & 0.102 & $\mathbf{0 . 0 0 2 2}$ \\
NLR & 0.711 & 0.0445 & $0.635-0.780$ & 0.123 & $\mathbf{0 . 0 0 0 7}$ \\
Y-value & 0.834 & 0.0315 & $0.768-0.887$ & - & - \\
\hline
\end{tabular}

Notes: ${ }^{a}$ AUC was calculated by subtracting the AUC of SUVmean or NLR from that of $Y$-value respectively
$[39,40]$. These insights shed new insights into the relationship between tumor metabolic activity and the host's inflammatory response process. The combination of these two types of parameters may serve as an effective predictor of treatment outcomes and prognoses. However, to date, we found only a few publications on this topic with cancer types such as intrahepatic cholangiocarcinoma [41], pancreatic cancer [42] and non-small cell lung cancer [43]. In the study of intrahepatic cholangiocarcinoma, researchers have developed a prognostic scoring system combining tumor SUVmax and NLR. The researchers assigned a prognostic score of 0 for patients with both low SUVmax and low NLR values, a score of 2 for patients with both high SUVmax and high NLR, and a score of 1 for the other patients. The researchers found significant differences in OS according to the prognostic scores. Similarly, the data from Shi S et al. [42] and St-Pierre Y et al. [43] proved that scoring systems that consider both metabolism parameters and inflammation parameters are able to stratify patients into different subgroups and are able to predict patient prognosis based on different scores. Although these studies demonstrate the predictive value of the combination of these two types of parameters, there were certain shortcomings in these studies. First, all of these studies simply scored patients as 0 or 1 based on the cut-off values of the metabolic and inflammatory indicators. These systems do not weigh the contribution of different indicators in predicting efficacy, which may lead to exaggerating or narrowing the role of a certain indicator. In addition, these systems do not include other factors that may affect prognosis. Second, these systems do not compare the performance of the scoring system with that of single indicators. In our study, we established a predictive model for treatment outcomes based on SUVmean and NLR in the training set that not only considers the contribution of different indicators but also includes other indicators that affect efficacy, i.e., tumor stage. Importantly, we verified the predictive performance of the model in the testing set and in all patients. Our data suggest that the accuracy of the prediction model is significantly better than that of the single SUVmean value or NLR value. With a cut-off value of 0.77 , the model has a high specificity and positive predictive value for predicting the treatment outcomes of EC patients treated with CCRT, although the model did not show an advantage in terms of sensitivity and negative predictive value. This model might therefore be able to identify patients who may be highly sensitive to CCRT and thus give these patients treatment with an appropriate intensity to avoid unnecessary adverse reactions. For patients who are not sensitive to CCRT, their treatment intensity and type of treatment may need to be tailored before treatment, thereby improving their underlying poor response to treatment.

Several limitations in the present study should to be mentioned. The first is its retrospective nature. And it is 


\begin{tabular}{|c|c|c|c|c|c|c|c|c|c|}
\hline \multirow[b]{3}{*}{$\mathrm{NLR} \leq \mathbf{2 . 4 2}$} & \multicolumn{2}{|c|}{ Training set } & & \multicolumn{2}{|c|}{ Testing set } & & \multicolumn{2}{|c|}{ All cases } & \\
\hline & OR & Ton-OR & & OR & n-OR & & OR & on-OR & \\
\hline & 37 & 6 & $\begin{array}{l}86.1 \% \\
\text { PPV }\end{array}$ & 29 & 5 & $\begin{array}{l}85.3 \% \\
\text { PPV }\end{array}$ & 66 & 11 & $\begin{array}{l}85.7 \% \\
\text { PPV }\end{array}$ \\
\hline NLR $>2.42$ & 19 & 18 & $\begin{array}{l}48.61 \% \\
\text { NPV }\end{array}$ & 27 & 22 & $\begin{array}{l}44.9 \% \\
\text { NPV }\end{array}$ & 46 & 40 & $\begin{array}{l}46.5 \% \\
\text { NPV }\end{array}$ \\
\hline & $\begin{array}{l}66.1 \% \\
\text { Sens }\end{array}$ & $\begin{array}{l}75.0 \% \\
\text { Spec }\end{array}$ & & $\begin{array}{l}51.8 \% \\
\text { Sens }\end{array}$ & $\begin{array}{l}81.5 \% \\
\text { Spec }\end{array}$ & & $\begin{array}{l}58.9 \% \\
\text { Sens }\end{array}$ & $\begin{array}{l}78.4 \% \\
\text { Spec }\end{array}$ & \\
\hline SUVmean $\leq 5.81$ & 39 & 6 & $\begin{array}{l}86.7 \% \\
\text { PPV }\end{array}$ & 36 & 8 & $\begin{array}{l}81.8 \% \\
\text { PPV }\end{array}$ & 75 & 14 & $\begin{array}{l}84.2 \% \\
\text { PPV }\end{array}$ \\
\hline SUVmean>5.81 & 17 & 18 & $\begin{array}{l}51.4 \% \\
\text { NPV }\end{array}$ & 20 & 19 & $\begin{array}{l}48.7 \% \\
\text { NPV }\end{array}$ & 37 & 37 & $\begin{array}{l}50.0 \% \\
\text { NPV }\end{array}$ \\
\hline & $\begin{array}{l}69.6 \% \\
\text { Sens }\end{array}$ & $\begin{array}{l}75.0 \% \\
\text { Spec }\end{array}$ & & $\begin{array}{l}64.3 \% \\
\text { Sens }\end{array}$ & $\begin{array}{l}70.4 \% \\
\text { Spec }\end{array}$ & & $\begin{array}{l}67.0 \% \\
\text { Sens }\end{array}$ & $\begin{array}{l}72.6 \% \\
\text { Spec }\end{array}$ & \\
\hline Y-value $>0.77$ & 34 & 1 & $\begin{array}{l}97.1 \% \\
\text { PPV }\end{array}$ & 35 & 3 & $\begin{array}{l}92.1 \% \\
\text { PPV }\end{array}$ & 69 & 4 & $\begin{array}{l}94.5 \% \\
\text { PPV }\end{array}$ \\
\hline Y-value $\leq 0.77$ & 22 & 23 & $\begin{array}{l}51.1 \% \\
\text { NPV }\end{array}$ & 21 & 24 & $\begin{array}{l}53.3 \% \\
\text { NPV }\end{array}$ & 43 & 47 & $\begin{array}{l}52.2 \% \\
\text { NPV }\end{array}$ \\
\hline & $\begin{array}{l}60.7 \% \\
\text { Sens }\end{array}$ & $\begin{array}{l}95.8 \% \\
\text { Spec }\end{array}$ & & $\begin{array}{l}62.5 \% \\
\text { Sens }\end{array}$ & $\begin{array}{l}88.9 \% \\
\text { Spec }\end{array}$ & & $\begin{array}{l}61.6 \% \\
\text { Sens }\end{array}$ & $\begin{array}{l}92.2 \% \\
\text { Spec }\end{array}$ & \\
\hline
\end{tabular}

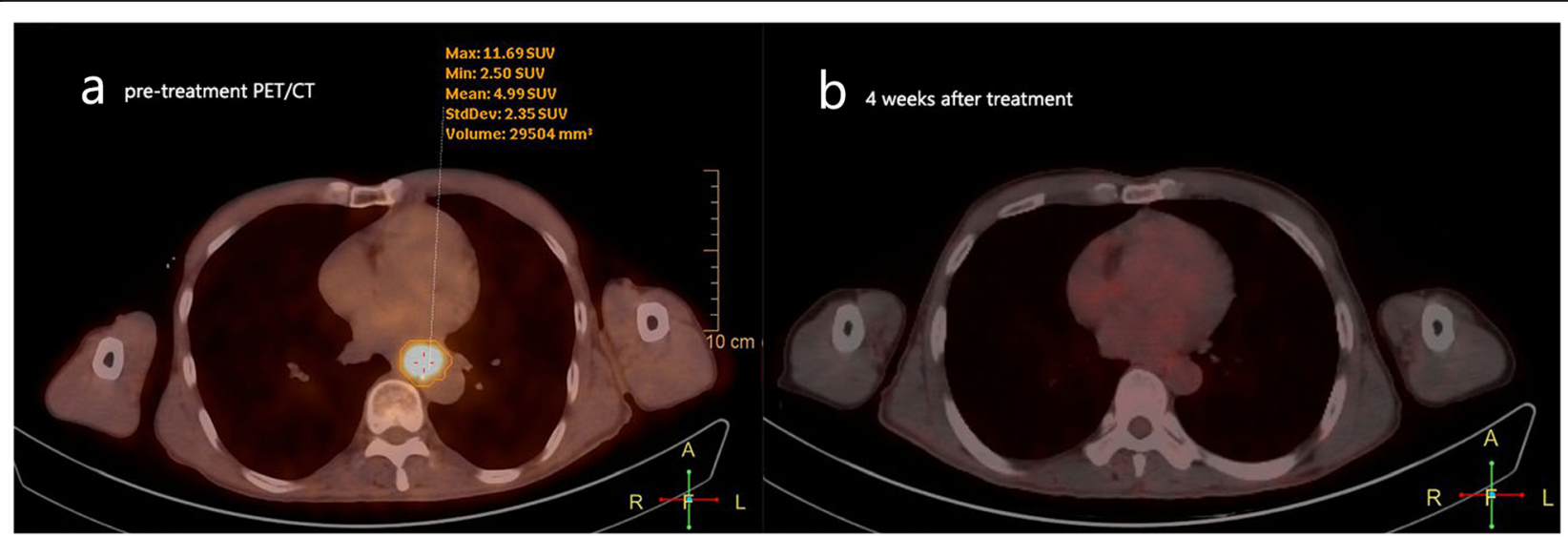

Fig. 5 This is a stage III ESCC patient with a SUVmean value at 4.99 and the NLR value at 0.80. According to the prediction model, the patient has Y-value of 0.86, which belongs to a good response patient. After receiving CCRT and sequential 2 cycles of chemotherapy, the repeated PET/CT examination showed that the primary esophageal lesion completely disappeared, and the treatment response was CR according to RECIST (version 1.1) evaluation standard 
a single center, small sample study. There are potential confounding factors that we cannot control. In the future, further prospective research should be conducted. Second, we do not have a clear explanation for the precise mechanism of the correlation between SUVmean and NLR. Finally, although we have demonstrated that this predictive model has a good performance in the testing set and in all patients, the model still needs to be verified by clinicians in practical work.

\section{Conclusion}

The pretreatment SUVmean and NLR values were independent predictors of treatment response to CCRT in patients with esophageal squamous cell carcinoma. The predictive model, which was constructed based on the pretreatment SUVmean and NLR values, provides a highly accurate tool for predicting patient outcomes after CCRT. This model may help clinicians identify subgroups of patients who are sensitive or insensitive to CCRT and to give these patients individualized and accurate treatment.

\section{Supplementary information}

Supplementary information accompanies this paper at https://doi.org/10. 1186/s12885-020-07040-8.

Additional file 1: Supplement Table 1. AUCS comparison of PET parameters

\section{Abbreviations \\ ${ }^{18}$ F-FDG PET/CT: ${ }^{18}$ F-fluorodeoxy-glucose positron emission tomography/ computed tomography; SUVmax: Maximum standardized uptake value; SUVmean: Mean standardized uptake value; NLR: Neutrophil to lymphocyte ratio; CCRT: Concurrent chemoradiotherapy; ROC: Receiver operating characteristic; AUC: Area under the curve; HR: Hazard ratio; ESCC: Esophageal squamous cell carcinoma; EC: Esophageal cancer; ECOG: Eastern Cooperative Oncology Group; IMRT: Intensity-modulated radiation therapy; RECIST: Response based on evaluation criteria in solid tumors; CR: Complete response; PR: Partial response; SD: Stable disease; PD: Progressive disease; OR: Objective response; ORR: Overall response rate}

\section{Acknowledgements}

Not applicable.

\section{Authors' contributions}

Conceptualization, MHL and YPS; Methodology, MHL and YPS; Software, YH and LM; Formal Analysis, CSW and KWZ; Resources, SLH; Data Curation, KWZ; Writing-Original Draft Preparation, CSW and KWZ; Writing-Review \& Editing, CSW and YPS; Supervision, MHL and YPS; Project Administration, MHL and YPS. All authors have read and approved the manuscript.

\section{Funding}

The Natural Science Foundation of China (NSFC 81672995), the National key research and development program (2018YFC1313201) supported this study.

\section{Availability of data and materials}

The datasets used and/or analyzed during the current study are available from the corresponding author on reasonable request.

\section{Ethics approval and consent to participate}

The ethics committee of Shandong Cancer Hospital and Institute approved the study. And informed consent was exempted due to the retrospective nature of the study.

\section{Consent for publication}

All authors have approved the manuscript and agree with submission to BMC cancer.

\section{Competing interests}

The authors declare that they have no competing interests.

\section{Author details}

'Department of Radiation Oncology, Qingdao University Medical College Affiliated Yantai Yuhuangding Hospital, 20 Yudong Road, Yantai 264000, Shandong, People's Republic of China. ${ }^{2}$ Department of Radiation Oncology, Shandong Cancer Hospital and Institute, Shandong University, 440 Jiyan Road, Jinan 250117, Shandong, People's Republic of China. ${ }^{3}$ Department of Nuclear Medicine, Shandong Cancer Hospital and Institute, Shandong University, 440 Jiyan Road, Jinan 250117, Shandong, People's Republic of China.

Received: 10 November 2019 Accepted: 4 June 2020

Published online: 10 June 2020

\section{References}

1. Cooper JS, Guo MD, Herskovic A, Macdonald JS, Martenson JA, Al-Sarraf M, et al. Chemoradiotherapy of locally advanced esophageal Cancer long-term follow-up of a prospective randomized trial (RTOG 85-01). J Am Med Assoc. 1999;281(17):1623-7

2. Zhao T, Chen H, Zhang T. Docetaxel and cisplatin concurrent with radiotherapy versus 5 -fluorouracil and cisplatin concurrent with radiotherapy in treatment for locally advanced oesophageal squamous cell carcinoma: a randomized clinical study. Med Oncol. 2012;29(5):3017-23.

3. Li QQ, Liu MZ, Hu YH, Liu H, He ZY, Lin HX. Definitive concomitant chemoradiotherapy with docetaxel and cisplatin in squamous esopheageal carcinoma. Dis Esophagus. 2010;23(3):253-9.

4. Zhu Y, Zhang W, Li Q, Li Q, Qiu B, Liu H, et al. A phase II randomized controlled trial: definitive concurrent Chemoradiotherapy with Docetaxel plus Cisplatin versus 5-fluorouracil plus Cisplatin in patients with Oesophageal squamous cell carcinoma. J Cancer. 2017:8(18):3657-66.

5. Yap JT, Carney JPJ, Hall NC, Townsend DW, Knoxville T. Image-guided cancer therapy using PET/CT. J Cancer. 2004;10(4):221-33.

6. Del Vecchio S, Zannetti A, Fonti R, lommelli F, Pizzuti LM, Lettieri A, et al. PET/CT in cancer research: from preclinical to clinical applications. Contrast Media Mol Imaging. 2010;5(4):190-200.

7. Hsu PP, Sabatini DM. Cancer cell metabolism: Warburg and beyond. Cell. 2008;134(5):703-7.

8. Miccò M, Vargas HA, Burger IA, Kollmeier MA, Goldman DA, Park KJ, et al. Combined pre-treatment MRI and 18F-FDG PET/CT parameters as prognostic biomarkers in patients with cervical cancer. Eur J Radiol. 2014; 83(7):1169-76.

9. Park GC, Kim JS, Roh JL, Choi SH, Nam SY, Kim SY. Prognostic value of metabolic tumor volume measured by 18F-FDG PET/CT in advanced-stage squamous cell carcinoma of the larynx and hypopharynx. Ann Oncol. 2012; 24(1):208-14.

10. Lin $P$, Min $M$, Lee $M$, Holloway $L$, Forstner $D$, Bray $V$, et al. Nodal parameters of FDG PET/CT performed during radiotherapy for locally advanced mucosal primary head and neck squamous cell carcinoma can predict treatment outcomes: SUVmean and response rate are useful imaging biomarkers. Eur J Nucl Med Mol Imaging. 2016;44(5):801-11.

11. Higgins KA, Hoang JK, Roach MC, Chino J, Yoo DS, Turkington TG, et al. Analysis of Pretreatment FDG-PET SUV Parameters in Head-and-Neck Cancer: Tumor SUVmean Has Superior Prognostic Value. Int J Rad Oncol*Biol*Phys. 2012;82(2):548-53.

12. Guler OC, Torun N, Yildirim BA, Onal C. Pretreatment metabolic tumour volume and total lesion glycolysis are not independent prognosticators for locally advanced cervical cancer patients treated with chemoradiotherapy. Br J Radiol. 2018;91(1084):20170552.

13. Hatt M, Van Stiphout R, Le Pogam A, Lammering G, Visvikis D, Lambin P. Early prediction of pathological response in locally advanced rectal cancer based on sequential18F-FDG PET. Acta Oncol. 2012;52(3):619-26.

14. Huang W, Zhou T, Ma L, Sun H, Gong H, Wang J, et al. Standard uptake value and metabolic tumor volume of 18F-FDG PET/CT predict short-term outcome early in the course of chemoradiotherapy in advanced non-small cell lung cancer. Eur J Nucl Med Mol Imaging. 2011;38(9):1628-35. 
15. Mantovani A, Allavena P, Sica A, Balkwill F. Cancer-related inflammation. Nature. 2008;454(7203):436-44.

16. Landskron G, De La Fuente M, Thuwajit P, Thuwajit C, Hermoso MA. Chronic inflammation and cytokines in the tumor microenvironment. J Immunol Res. 2014;2014:1-19.

17. Elinav E, Nowarski R, Thaiss CA, Hu B, Jin C, Flavell RA. Inflammation-induced cancer: crosstalk between tumours, immune cells and microorganisms. Nat Rev Cancer. 2013;13(11):759-71.

18. Sharaiha RZ, Halazun KJ, Mirza F, Port JL, Lee PC, Neugut Al, et al. Elevated preoperative neutrophil:lymphocyte ratio as a predictor of postoperative disease recurrence in esophageal Cancer. Ann Surg Oncol. 2011;18(12): 3362-9.

19. Sato $H$, Tsubosa $Y$, Kawano T. Correlation between the Pretherapeutic neutrophil to lymphocyte ratio and the pathologic response to Neoadjuvant chemotherapy in patients with advanced esophageal Cancer. World J Surg. 2012;36(3):617-22.

20. Yoo EJ, Park JC, Kim EH, Park CH, Shim CN, Lee HJ, et al. Prognostic value of neutrophil-to-lymphocyte ratio in patients treated with concurrent chemoradiotherapy for locally advanced oesophageal cancer. Dig Liver Dis. 2014;46(9):846-53.

21. Liu X, Li M, Zhao F, Zhu Y, Luo Y, Kong L, et al. The lymphocyte\&ndash; monocyte ratio predicts tumor response and survival in patients with locally advanced esophageal cancer who received definitive chemoradiotherapy. OncoTargets Ther. 2017;10:871-7.

22. Azimi F, Scolyer RA, Rumcheva P, Moncrieff M, Murali R, Mccarthy SW, et al. Tumor-infiltrating lymphocyte grade is an independent predictor of sentinel lymph node status and survival in patients with cutaneous melanoma. J Clin Oncol. 2012;30(21):2678-83.

23. Sa R. Progress in human tumour immunology and immunotherapy. Nature. 2001:17(411):380-4.

24. Mcsorley ST, Khor BY, Tsang K, Colville D, Han S, Horgan PG, et al. The relationship between 18F-FDG-PETCT-derived markers of tumour metabolism and systemic inflammation in patients with recurrent disease following surgery for colorectal cancer. Color Dis. 2018;20(5):407-15.

25. Ino Y, Yamazaki-Itoh R, Shimada K, Iwasaki M, Kosuge T, Kanai Y, et al. Immune cell infiltration as an indicator of the immune microenvironment of pancreatic cancer. Br J Cancer. 2013;108(4):914-23.

26. Stotz M, Gerger A, Eisner F, Szkandera J, Loibner H, Ress AL, et al. Increased neutrophil-lymphocyte ratio is a poor prognostic factor in patients with primary operable and inoperable pancreatic cancer. Br J Cancer. 109(2, 2013):416-21

27. Prabawa IPY, Bharghah A, Liwang F, Tandio DA, Tandio AL, AaW L, et al. Pretreatment Neutrophil-to-Lymphocyte ratio (NLR) and Platelet-toLymphocyte Ratio (PLR) as a Predictive Value of Hematological Markers in Cervical Cancer. Asian Pac J Cancer Prev. 2019;20(3):863-8.

28. Miyamoto R, Inagawa S, Sano N, Tadano S, Adachi S, Yamamoto M. The neutrophil-to-lymphocyte ratio (NLR) predicts short-term and long-term outcomes in gastric cancer patients. Eur J Surg Oncol. 2018;44(5):607-12.

29. Kosumi K, Baba Y, Ishimoto T, Harada K, Nakamura K, Ohuchi M, et al. Neutrophil/lymphocyte ratio predicts the prognosis in esophageal squamous cell carcinoma patients. Surg Today. 2015;46(4):405-13.

30. Rashid F, Waraich N, Bhatti I, Saha S, Khan RN, Ahmed J, et al. A preoperative elevated neutrophil: lymphocyte ratio does not predict survival from oesophageal cancer resection. World J Surg Oncol. 2010;8(1).

31. Dutta S, Fullarton GM, Mcmillan DC, Crumley ABC, Horgan PG. Comparison of the prognostic value of tumour-and patient-related factors in patients undergoing potentially curative resection of oesophageal cancer. World J Surg. 2011;35(8):1861-6.

32. Fujii T, Yanai K, Tokuda S, Nakazawa Y, Kurozumi S, Obayashi S, et al. Relationship between FDG uptake and neutrophil/lymphocyte ratio in patients with invasive ductal breast Cancer. Anticancer Res. 2018;38(8):4927-31.

33. Jeong E, Hyun SH, Moon SH, Cho YS, Kim B-T, Lee K-H. Relation between tumor FDG uptake and hematologic prognostic indicators in stage I lung cancer patients following curative resection. Medicine. 2017;96(5):e5935.

34. Du S, Sun H, Gao S, Xin J, Lu Z. Metabolic parameters with different thresholds for evaluating tumor recurrence and their correlations with hematological parameters in locally advanced squamous cell cervical carcinoma: an observational 18F-FDG PET/CT study. Quant Imaging Med Surg. 2019;9(3):440-52.

35. Xu J, Li Y, Hu S, Lu L, Gao Z, Yuan H. The significant value of predicting prognosis in patients with colorectal cancer using 18F-FDG PET metabolic parameters of primary tumors and hematological parameters. Ann Nucl Med. 2018;33(1):32-8.

36. Sürücü $E$, Demir $Y$, Şengöz $T$. The correlation between the metabolic tumor volume and hematological parameters in patients with esophageal cancer. Ann Nucl Med. 2015;29(10):906-10.

37. Mirili C, Guney IB, Paydas S, Seydaoglu G, Kapukaya TK, Ogul A, et al. Prognostic significance of neutrophil/lymphocyte ratio (NLR) and correlation with PET-CT metabolic parameters in small cell lung cancer (SCLC). Int J Clin Oncol. 2018;24(2):168-78.

38. Kubota R, Yamada S, Kubota K, Ishiwata K, Tamahashi N, Ido T. Intratumoral distribution of fluorine-18-fluorodeoxyglucose in vivo: high accumulation in macrophages and granulation tissues studied by microautoradiography. J Nucl Med. 1992;33(11):1972-80.

39. Clavo AC, Brown RS, Wahl RL. Fluorodeoxyglucose uptake in human cancer cell lines is increased by hypoxia. J Nucl Med. 1995;36(9):1625-32.

40. Kwilas AR, Donahue RN, Tsang KY, Hodge JW. Immune consequences of tyrosine kinase inhibitors that synergize with Cancer immunotherapy. Cancer Cell Microenvironment. 2015;2(1):e677.

41. Seo S, Yoh T, Morino K, Fuji H, Taura K, Fukumitsu KEN, et al. The relationship between 18F-FDG uptake on PET/CT and markers of systemic inflammatory response in patients undergoing surgery for intrahepatic Cholangiocarcinoma. Anticancer Res. 2019;39(1):341-6.

42. Shi $S, Y e L, Z$ hao Q, Hu Y, Huang Y, Chen G, et al. Prognostic index score predicts outcome of patients with stage I non-small cell lung cancer after stereotactic body radiation therapy. Jpn J Clin Oncol. 2019;49(4):367-72.

43. St-Pierre $Y$, Choi $Y$, Oh D-Y, Park H, Kim T-Y, Lee K-H, et al. More accurate prediction of metastatic pancreatic Cancer patients' survival with prognostic model using both host immunity and tumor metabolic activity. PLoS One. 2016;11(1):e0145692

\section{Publisher's Note}

Springer Nature remains neutral with regard to jurisdictional claims in published maps and institutional affiliations.

\section{Ready to submit your research? Choose BMC and benefit from:}

- fast, convenient online submission

- thorough peer review by experienced researchers in your field

- rapid publication on acceptance

- support for research data, including large and complex data types

- gold Open Access which fosters wider collaboration and increased citations

- maximum visibility for your research: over $100 \mathrm{M}$ website views per year

At $\mathrm{BMC}$, research is always in progress.

Learn more biomedcentral.com/submissions 